\title{
AN EXAMPLE OF A BOUNDED C-CONVEX DOMAIN WHICH IS NOT BIHOLOMORPHIC TO A CONVEX DOMAIN
}

\author{
NIKOLAI NIKOLOV, PETER PFLUG and WŁODZIMIERZ ZWONEK*
}

\begin{abstract}
We show that the symmetrized bidisc is a C-convex domain. This provides an example of a bounded C-convex domain which cannot be exhausted by domains biholomorphic to convex domains.
\end{abstract}

\section{Introduction}

Recall that a domain $D$ in $\mathrm{C}^{n}$ is called C-convex if any non-empty intersection with a complex line is contractible (cf. [2], [9]). A consequence of the fundamental Lempert theorem (see [12]) is the fact that any bounded C-convex domain $D$ with $C^{2}$ boundary has the following property (see [8]):

\section{(*) The Carathéodory distance and the Lempert function of D coincide.}

Any convex domain can be exhausted by smooth bounded convex ones (which are obviously C-convex); therefore, any convex domain satisfies $(*)$, too. To extend this phenomenon to bounded C-convex domains (see Problem 4' in [14]), it is sufficient to give a positive answer to one of the following questions:

(a) Can any bounded $\mathrm{C}$-convex domain be exhausted by $C^{2}$-smooth $\mathrm{C}$-convex domains? (See Problem 2 in [14] and Remark 2.5.20 in [2].)

(b) Is any bounded C-convex domain biholomorphic to a convex domain? (See Problem 4 in [14].)

The main aim of this note is to give a negative answer to the question (b).

Denote by $\mathrm{G}_{2}$ the so-called symmetrized bidisc, that is, the image of the bidisc under the mapping whose components are the two elementary symmetric functions of two complex variables. $G_{2}$ serves as the first example of a

\footnotetext{
* The paper was initiated while the third author's research stay at the Carl von Ossietzky Universität Oldenburg which was supported by the Alexander von Humboldt Foundation. The thirdnamed author was also supported by the KBN Research Grant No. 1 PO3A 005 28. The first and second named authors were supported by grants from DFG (DFG-Projekt 227/8).

Received September 25, 2006.
} 
bounded pseudoconvex domain in $\mathrm{C}^{2}$ with the property $(*)$ which cannot be exhausted by domains biholomorphic to convex domains (see [3], [6]). We shall show that $\mathrm{G}_{2}$ is a $\mathrm{C}$-convex domain. This fact gives a counterexample to the question (b) and simultaneously, it supports the conjecture that (cf. Problem 4' in [14]) any bounded C-convex domain has property (*). Note that the answer to the question (a) for $G_{2}$ is not known. The positive answer to this question would imply an alternative (to that of [4] and [1]) proof of the equality of the Carathéodory distance and Lempert function on $\mathrm{G}_{2}$ whereas the negative answer would solve Problem 2 in [14].

Some additional properties of C-convex domains and symmetrized polydiscs are also given in the paper.

\section{Background and results}

Recall that a domain $D$ in $C^{n}$ is called (cf. [9], [2]):

- C-convex if any non-empty intersection with a complex line is contractible (i.e. $D \cap L$ is connected and simply connected for any complex affine line $L$ such that $L \cap D$ is not empty);

- linearly convex if its complement in $\mathrm{C}^{n}$ is a union of affine complex hyperplanes;

- weakly linearly convex if for any $a \in \partial D$ there exists an affine complex hyperplane through $a$ which does not intersect $D$.

Note that the following implications hold:

$$
\text { C-convexity } \Rightarrow \text { linear convexity } \Rightarrow \text { weak linear convexity. }
$$

Moreover, these three notions coincide in the case of bounded domains with $C^{1}$ boundary (cf. [2], [9]).

Let $\mathrm{D}$ denote the unit disc in $\mathrm{C}$. Let $\pi_{n}=\left(\pi_{n, 1}, \ldots, \pi_{n, n}\right): \mathrm{C}^{n} \rightarrow \mathrm{C}^{n}$ be defined as follows:

$$
\pi_{n, k}(\mu)=\sum_{1 \leq j_{1}<\cdots<j_{k} \leq n} \mu_{j_{1}} \ldots, \mu_{j_{k}}, \quad 1 \leq k \leq n, \quad \mu=\left(\mu_{1}, \ldots, \mu_{n}\right) \in \mathrm{C}^{n} .
$$

The set $\mathrm{G}_{n}:=\pi_{n}\left(\mathrm{D}^{n}\right)$ is called the symmetrized $n$-disc (cf. [1], [11]).

Recall that $\mathrm{G}_{2}$ is the first example of a bounded pseudoconvex domain with the property $(*)$, which cannot be exhausted by domains biholomorphic to convex ones (see [3], [6]). On the other hand, $\mathrm{G}_{n}, n \geq 3$, does not satisfy the property $(*)$ (see [13]). In particular, it cannot be exhausted by domains biholomorphic to convex domains, either. 
In this note we shall show the following additional properties of domains $\mathrm{G}_{n}, n \geq 2$.

Theorem 1. (i) $\mathrm{G}_{2}$ is a $\mathrm{C}$-convex domain.

(ii) $\mathrm{G}_{n}, n \geq 3$, is a linearly convex domain which is not $\mathrm{C}$-convex.

Theorem 1 (i) together with a result of [3] and [6] gives a negative answer to the following question posed by S. V. Znamenskii (cf. Problem 4 in [14]):

Is any bounded $\mathrm{C}$-convex domain biholomorphic to a convex domain?

Moreover, it seems to us that Theorem 1 (ii) gives the first example of a linearly convex domain homeomorphic to $\mathrm{C}^{n}, n \geq 3$, which is not $\mathrm{C}$-convex, is not a Cartesian product and does not satisfy property $(*)$. To see that $\mathrm{G}_{n}$ is homeomorphic to $\mathbf{C}^{n}$, observe that $\rho_{\lambda}(z):=\left(\lambda z_{1}, \lambda^{2} z_{2}, \ldots, \lambda^{n} z_{n}\right) \in \mathbf{G}_{n}$ if $z \in \mathrm{G}_{n}$ and $\lambda \in \mathrm{C}$. Then setting $h(z)=\max _{1 \leq j \leq n}\left\{\left|\mu_{j}\right|: \pi_{n}(\mu)=z\right\}$ and $g(z)=\frac{1}{1-h(z)}$, it is easy to see that the function $\mathrm{G}_{n} \ni z \mapsto \rho_{g(z)}(z) \in \mathrm{C}^{n}$ is the desired homeomorphism.

These remarks also show that $\mathrm{G}_{n}$ is close, in some sense, to a balanced domain, that is, a domain $D$ in $\mathrm{C}^{n}$ such that $\lambda z \in D$ for any $z \in D$ and $\lambda \in \overline{\mathrm{D}}$. On the other hand, in spite of the properties of $\mathrm{G}_{n}$, one has the following.

Proposition 2. Any weakly linearly convex balanced domain is convex.

This proposition is a simple extension of Example 2.2.4 in [2], where it is shown that any C-convex complete Reinhardt domain is convex.

We may also prove some general property of C-convex domains showing that all non-degenerate $\mathrm{C}$-convex domains, that is, containing no complex lines, are $c$-finitely compact. For definitions of the Carathéodory distance $c_{D}$ of the domain $D, c$-finite compactness, $c$-completeness and basic properties of these notions we refer the Reader to consult [10].

Observe that a degenerate linearly convex domain $D$ is linearly equivalent to $C \times D^{\prime}$ (cf. Proposition 4.6.11 in [9]). Indeed, we may assume that $D$ contains the $z_{1}$-line. Since the complement ${ }^{c} D$ of $D$ is a union of complex hyperplanes disjoint from this line, then ${ }^{c} D=\mathrm{C} \times G$ and hence $D=\mathrm{C} \times{ }^{c} G$. On the other hand, we have

Proposition 3. Any non-degenerate C-convex domain is biholomorphic to a bounded domain and c-finitely compact. In particular, it is c-complete and hyperconvex.

REMARKs. (i) In virtue of Proposition 3, we claim that one may conjecture more than the question (a) (see [15]), namely, any C-convex domain containing no complex hyperplanes can be exhausted by bounded $C^{2}$-smooth C-convex 
domains (this is not true in general without the above assumption); then the Carathéodory pseudodistance and Lempert function will coincide on any Cconvex domain.

(ii) The hyperconvexity of $\mathrm{G}_{n}$ is simple and well-known (see [7]). The above proposition implies more in dimension two. Namely, it implies that the symmetrized bidisc is $c$-finitely compact. Although the symmetrized polydiscs in higher dimensions are not $\mathrm{C}$-convex the conclusion of the above proposition, that is, the $c$-finite compactness of the symmetrized $n$-disc $\mathrm{G}_{n}$, holds for any $n \geq 2$. In fact, it is a straightforward consequence of Corollary 3.2 in [5].

(iii) Finally, we mention that, for $n \geq 2, \mathrm{G}_{n}$ is starlike with respect to the origin if and only if $n=2$. This observation gives the next difference in the geometric shape of the 2-dimensional and higher dimensional symmetrized discs. Recall that the fact that $\mathrm{G}_{2}$ is starlike is contained in [1]. For the converse just take the point $(3,3,1,0, \ldots, 0)$.

\section{Proofs}

Proof of Theorem 1 (i). We shall make use of the following description of C-convex domains. For $a \in \partial D$, denote by $\Gamma(a)$ the set of all hyperplanes through $a$ and disjoint from $D$. Then a bounded domain $D$ in $\mathrm{C}^{n}, n>1$, is Cconvex if and only if for any $a \in \partial D$ the set $\Gamma(a)$ is non-empty and connected as a set in $\mathrm{CP}^{n}$ (cf. Theorem 2.5.2 in [2]).

So we have to check that $\Gamma(a)$ is non-empty and connected for any $a \in \partial \mathbf{G}_{2}$.

Let us first consider a regular point of $\partial \mathrm{G}_{2}$, that is, a point of the form $\pi_{2}(\mu)$, where $\left|\mu_{1}\right|=1,\left|\mu_{2}\right|<1$ (or vice versa). Then the complex tangent line to $\partial D$ at $a$ is of the form $\left\{\pi_{2}\left(\mu_{1}, \lambda\right): \lambda \in \mathrm{C}\right\}$, which is obviously disjoint from $\mathrm{G}_{2}$. So $\Gamma(a)$ is a singleton.

Now we fix a non-regular point of $\partial \mathrm{G}_{2}$, that is, a point of the form $\pi_{2}(\mu)$, where $\left|\mu_{1}\right|=\left|\mu_{2}\right|=1$.

After a rotation we may assume that $\mu_{1} \mu_{2}=1$, that is, $\mu_{2}=\bar{\mu}_{1}$. Then $\mu_{1}+\mu_{2}=2 \operatorname{Re} \mu_{1}=: 2 x$, where $x \in[-1,1]$.

We shall find all the possible directions of complex lines passing simultaneously through $\pi_{2}(\mu)$ and an element of $G_{2}$. Any such line is of the form $\pi_{2}(\mu)+\mathrm{C}\left(\pi_{2}(\mu)-\pi_{2}(\lambda)\right)$, where $\lambda \in \mathrm{D}^{2}$. So

$$
A:={ }^{c} \Gamma\left(\pi_{2}(\mu)\right)=\left\{\frac{\lambda_{1}+\lambda_{2}-2 x}{\lambda_{1} \lambda_{2}-1}: \lambda_{1}, \lambda_{2} \in \mathrm{D}\right\} .
$$

In particular, $\Gamma\left(\pi_{2}(\mu)\right) \neq \emptyset$.

To show the connectedness of $\Gamma\left(\pi_{2}(\mu)\right)$, we shall check the simple-connectedness of $A$. Let us recall that the mapping $\frac{z-\alpha}{z-\beta}$, where $|\beta|>1$, maps the 
unit disc $\mathrm{D}$ onto the disc $\triangle\left(\frac{1-\alpha \bar{\beta}}{1-|\beta|^{2}}, \frac{|\alpha-\beta|}{|\beta|^{2}-1}\right)$, so

$$
\left\{\frac{\lambda+\lambda_{1}-2 x}{\lambda \lambda_{1}-1}: \lambda \in \mathrm{D}\right\}=\Delta\left(\frac{2 x-2 \operatorname{Re} \lambda_{1}}{1-\left|\lambda_{1}\right|^{2}}, \frac{\left|2 x \lambda_{1}-\lambda_{1}^{2}-1\right|}{1-\left|\lambda_{1}\right|^{2}}\right)=: A_{\lambda_{1}} .
$$

Consequently the set $A=\bigcup_{\lambda_{1} \in \mathrm{D}} A_{\lambda_{1}} \subset \mathrm{C}$ is simply connected.

Proof of Theorem 1 (ii). For the proof of the linear convexity of $\mathbf{G}_{n}$ consider the point $z=\pi_{n}(\lambda) \in \mathrm{C}^{n} \backslash \mathrm{G}_{n}$. We may assume that $\left|\lambda_{1}\right| \geq 1$. Then the set

$$
B:=\left\{\pi_{n}\left(\lambda_{1}, \mu_{1}, \ldots, \mu_{n-1}\right): \mu_{1}, \ldots, \mu_{n-1} \in \mathrm{C}\right\}
$$

is disjoint from $\mathrm{G}_{n}$. On the other hand, it is easy to see that

$$
B=\left\{\left(\lambda_{1}+z_{1}, \lambda_{1} z_{1}+z_{2}, \ldots, \lambda_{1} z_{n-2}+z_{n-1}, \lambda_{1} z_{n-1}\right): z_{1}, \ldots, z_{n-1} \in \mathrm{C}\right\},
$$

so $B$ is a complex affine hyperplane. Hence $\mathrm{G}_{n}$ is linearly convex.

To show that $\mathrm{G}_{n}$ is not $\mathrm{C}$-convex for $n \geq 3$, consider the points

$$
\begin{aligned}
& a_{t}:=\pi_{n}(t, t, t, 0, \ldots, 0)=\left(3 t, 3 t^{2}, t^{3}, 0, \ldots, 0\right), \\
& b_{t}:=\pi_{n}(-t,-t,-t, 0, \ldots, 0)=\left(-3 t, 3 t^{2},-t^{3}, 0, \ldots, 0\right), \quad t \in(0,1) .
\end{aligned}
$$

Obviously $a_{t}, b_{t} \in \mathrm{G}_{n}$. Denote by $L_{t}$ the complex line passing through $a_{t}$ and $b_{t}$, that is,

$$
L_{t}=\left\{c_{t, \lambda}:=\left(3 t(1-2 \lambda), 3 t^{2}, t^{3}(1-2 \lambda), 0, \ldots, 0\right): \lambda \in \mathrm{C}\right\} .
$$

Assume that the set $\mathrm{G}_{n} \cap L_{t}$ is connected. Since $a_{t}=c_{t, 0}$ and $b_{t}=c_{t, 1}$, then $c_{t, \lambda} \in \mathrm{G}_{n}$ for some $\lambda=\frac{1}{2}+i \tau, \tau \in \mathrm{R}$. It follows that

$$
c_{t, \lambda}=\left(-6 i \tau t, 3 t^{2},-2 i \tau t^{3}, 0, \ldots, 0\right) .
$$

We may choose $\mu \in \mathrm{D}^{n}$ such that $\mu_{j}=0, j=4, \ldots, n$, and $c_{t, \lambda}=\pi_{n}(\mu)$, $\mu \in \mathrm{D}^{n}$. Then $-36 \tau^{2} t^{2}=\left(\mu_{1}+\mu_{2}+\mu_{3}\right)^{2}=\mu_{1}^{2}+\mu_{2}^{2}+\mu_{3}^{2}+6 t^{2}$ and hence

$$
t^{2}=\frac{\left|\mu_{1}^{2}+\mu_{2}^{2}+\mu_{3}^{2}\right|}{36 \tau^{2}+6}<\frac{3}{36 \tau^{2}+6} \leq \frac{1}{2} .
$$

Therefore, $\mathrm{G}_{n} \cap L_{t}$ is not connected if $t \in\left[\frac{1}{\sqrt{2}}, 1\right)$ and so $\mathrm{G}_{n}$ is not a $\mathrm{C}$-convex domain.

Proof of Proposition 2. Set $D^{*}:=\left\{w \in C^{n}:<z, w>\neq 1, \forall z \in\right.$ $D$ \}. We shall use the fact that a domain $D$ in $C^{n}$ containing the origin is weakly linearly convex if and only if $D$ is a connected component of $D^{* *}$ (cf. Proposition 2.1.4 in [2]). 
Since our domain $D$ is balanced, it is easy to see that $D^{*}$ is balanced. We shall show $D^{*}$ is convex. Then, applying this fact to $D^{*}$, we conclude that $D^{* *}$ is a convex balanced domain. On the other hand, it follows by our assumption that $D$ is a component of $D^{* *}$ and hence $D^{* *}=D$.

To see that $D^{*}$ is convex, suppose the contrary. Then we find points $w_{1}, w_{2} \in$ $D^{*}, z \in D$ and a number $t \in(0,1)$ such that $\left\langle z, t w_{1}+(1-t) w_{2}\right\rangle=1$. We may assume that $\left|\left\langle z, w_{1}\right\rangle\right| \geq 1$. Since $D$ is balanced, we get $\tilde{z}:=\frac{z}{\left\langle z, w_{1}\right\rangle} \in D$ and $\left\langle\tilde{z}, w_{1}\right\rangle=1$, a contradiction.

Proof of Proposition 3. Let $D$ be non-degenerate C-convex domain in $\mathrm{C}^{n}$. For any point $z \in{ }^{c} D$ consider a hyperplane $L_{z}$ through $z$ and disjoint from $D$. Let $l_{z}$ be the orthogonal line through 0 and orthogonal to $L_{z}$. Denote by $\pi_{z}$ the orthogonal projection of $\mathrm{C}^{n}$ onto $l_{z}$ and set $a_{z}=\pi_{z}(a)$. Observe that $D_{z}=\pi_{z}(D)$ is biholomorphic to $\mathrm{D}$, since it is connected, simply connected (cf. Theorem 2.3.6 in [2]) and $\pi_{z}(z) \notin \pi_{z}(D)$. Moreover, since $D$ is a non-degenerate linearly convex domain, it is easy to see that there are $n$ C-independent $l_{z}$ 's. We may assume that these $l_{z}$ 's are the set $C$ of coordinate planes. Then $D \subset G:=\prod_{l_{z} \in C} \pi_{z}(D)$ and $G$ is biholomorphic to the polydisc $\mathrm{D}^{n}$. In particular, $D$ is biholomorphic to a bounded domain, hence it is $c$-hyperbolic.

Further, we may assume that $0 \in D$. To see that $D$ is $c$-finitely compact, it is enough to show that $\lim _{a \rightarrow z} c_{D}(0 ; a)=\infty$ for any $z \in \partial D$ and, if $D$ is unbounded, $z=\infty$. But the last one follows by the fact that $G$ is $c$-finitely compact. On the other hand, if $a \rightarrow z \in \partial D$, then $a_{z} \rightarrow \pi_{z}(z) \in \partial D_{z}$ and hence $c_{D}(0 ; a) \geq c_{D_{z}}\left(0 ; a_{z}\right) \rightarrow \infty$.

\section{REFERENCES}

1. Agler, J., Young, N. J., The hyperbolic geometry of the symmetrized bidisc, J. Geom. Anal. 14 (2004), 375-403.

2. Andersson, M., Passare, M., Sigurdsson, R., Complex Convexity and Analytic Functionals, Birkhäuser, Basel-Boston-Berlin, 2004.

3. Costara, C., The symmetrized bidisc and Lempert's theorem, Bull. London Math. Soc. 36 (2004), 656-662.

4. Costara, C., Dissertation, Université Laval (2004).

5. Costara, C., On the spectral Nevanlinna-Pick problem, Studia Math. 170 (2005), 23-55.

6. Edigarian, A., A note on Costara's paper, Ann. Polon. Math. 83 (2004), 189-191.

7. Edigarian, A., Zwonek, W., Geometry of the symmetrized polydisc, Arch. Math. (Basel) 84 (2005), 364-374.

8. Jacquet, D., C-convex domains with $C^{2}$ boundary, Complex Var. Elliptic Equ. 51 (2006), 303-312.

9. Hörmander, L., Notions of Convexity, Birkhäuser, Basel-Boston-Berlin, 1994.

10. Jarnicki, M., Pflug, P., Invariant Distances and Metrics in Complex Analysis, de Gruyter Exp. Math. 8 (1993). 
11. Jarnicki, M., Pflug, P., Invariant distances and metrics in complex analysis - revisited, Dissertationes Math. (Rozprawy Mat.) 430 (2005), 1-192.

12. Lempert, L., La métrique de Kobayashi et la représentation des domaines sur la boule, Bull. Soc. Math. France 109 (1981), 427-474.

13. Nikolov, N., Pflug, P., Zwonek, W., The Lempert function of the symmetrized polydisc in higher dimensions is not a distance, Proc. Amer. Math. Soc. 135 (2007), 2921-2928.

14. Znamenskii, S. V., Seven C-convexity problems (in Russian), Complex analysis in modern mathematics. On the 80th anniversary of the birth of Boris Vladimirovich Shabat, E. M. Chirka (ed.), FAZIS, Moscow, 2001, 123-131.

15. Znamenskii, S. V., Znamenskaya, L. N., Spiral connectedness of the sections and projections of C-convex sets, Math. Notes 59 (1996), 253-260.

INSTITUTE OF MATHEMATICS AND INFORMATICS BULGARIAN ACADEMY OF SCIENCES

1113 SOFIA

BULGARIA

E-mail: nik@math.bas.bg

INSTYTUT MATEMATYKI

UNIWERSYTET JAGIELLOŃSKI

REYMONTA 4

30-059 KRAKÓW

POLAND

E-mail: Wlodzimierz.Zwonek@im.uj.edu.pl
CARL VON OSSIETZKY UNIVERSITÄT OLDENBURG INSTITUT FÜR MATHEMATIK

POSTFACH 2503

D-26111 OLDENBURG

GERMANY

E-mail: pflug@mathematik.uni-oldenburg.de 